\title{
Veno-venous extracorporeal membrane oxygenation in the surgical management of post-traumatic intrathoracic tracheal transection
}

\author{
Angelo Carretta ${ }^{1}$, Paola Ciriaco ${ }^{1}$, Alessandro Bandiera ${ }^{1}$, Piergiorgio Muriana ${ }^{1}$, Federico Pappalardo ${ }^{2}$, \\ Lars Mikael Broman ${ }^{3}$, Andrea Montisci ${ }^{4}$, Giampiero Negri ${ }^{1}$ \\ ${ }^{1}$ Department of Thoracic Surgery, San Raffaele Hospital, Vita Salute San Raffaele University, Milan, Italy; ${ }^{2}$ Department of Cardiothoracic Anesthesia \\ and Intensive Care, Advanced Heart Failure and Mechanical Circulatory Support Program, San Raffaele Hospital, Vita Salute San Raffaele \\ University, Milan, Italy; ${ }^{3}$ ECMO Centre Karolinska, Department of Pediatric Perioperative Medicine and Intensive Care, Karolinska University \\ Hospital, and Department of Physiology and Pharmacology, Karolinska Institutet, Stockholm, Sweden; ${ }^{4}$ Department of Anesthesia and Intensive \\ Care, Cardiothoracic Centre, Istituto Clinico Sant'Ambrogio, Gruppo Ospedaliero San Donato, Milan, Italy \\ Correspondence to: Angelo Carretta, MD. Department of Thoracic Surgery, San Raffaele Hospital, Vita Salute San Raffaele University, Via Olgettina, \\ 60 - 20132 Milan, Italy. Email: angelo.carretta@hsr.it.
}

Submitted Jun 01, 2018. Accepted for publication Nov 20, 2018.

doi: $10.21037 /$ jtd.2018.11.117

View this article at: http://dx.doi.org/10.21037/jtd.2018.11.117

\section{Introduction}

Complete traumatic blunt tracheal transection is a rare but life-threatening event, and urgent tracheal repair is required to restore ventilation (1). Emergent intubation of the distal tracheal stump bridging the lesion by means of tracheostomy or under bronchoscopic guidance is usually required to restore adequate gas exchange. However, especially in case of lesions of the intrathoracic trachea, intubation across the transected stumps of the trachea is not always possible due to the risk of causing a migration of the distal segment into the mediastinum, thus completely losing control of ventilation. Whenever intubation of the distal tracheal stump is not possible, and the lesion cannot be reached by means of a tracheostomy, possible options to restore adequate gas exchange consist of emergent surgical treatment and use of cardiopulmonary bypass (CPB) (2) or extracorporeal membrane oxygenation (ECMO) (3).

\section{Case presentation}

A 36-year-old man was involved in a high-speed car accident; after fluid resuscitation and unsuccessful attempt of endotracheal intubation on the field, he was brought to the Emergency Department of our hospital. On arrival he was still conscious, hypoxic $\left(\mathrm{SaO}_{2} 74 \%\right)$ with evidence of subcutaneous emphysema at the neck and upper thorax. A chest X-ray showed a left pneumothorax and a chest tube was inserted. A laryngeal mask was positioned restoring adequate gas exchange and intubation through the laryngeal mask under bronchoscopic control was attempted. Bronchoscopy showed a complete separation of the intrathoracic trachea. Translesional intubation under bronchoscopic assistance was not completed due to collapse of mediastinal space during the procedure and to the risk of causing a further migration of the distal tracheal stump into the mediastinum. CT scan confirmed a complete separation of the intrathoracic trachea $3 \mathrm{~cm}$ above the carina (Figure 1), associated with extensive bilateral lung contusion, mediastinal emphysema and multiple bilateral rib fractures. Urgent tracheal repair was planned.

\section{Treatment and follow-up}

Due to the impossibility of achieving translesional intubation of the distal stump, ECMO was started before surgery. A femoro-femoral cannulation approach was chosen due to the difficulties in approaching the neck for cannula implantation related to the presence of the surgical incision. Transesophageal echocardiography was also deferred due to the risk of dislodging the tracheal stumps with the introduction of the ultrasound probe. Under 


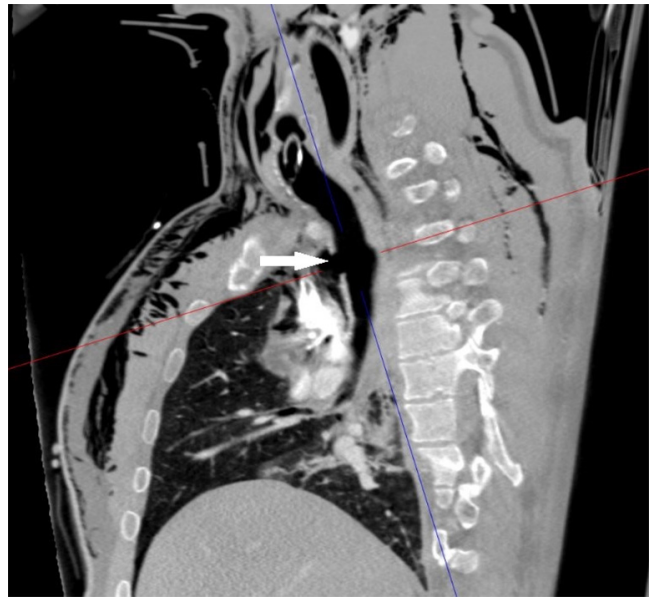

Figure 1 CT scan, sagittal reconstruction: complete transection of the intrathoracic trachea (arrow).

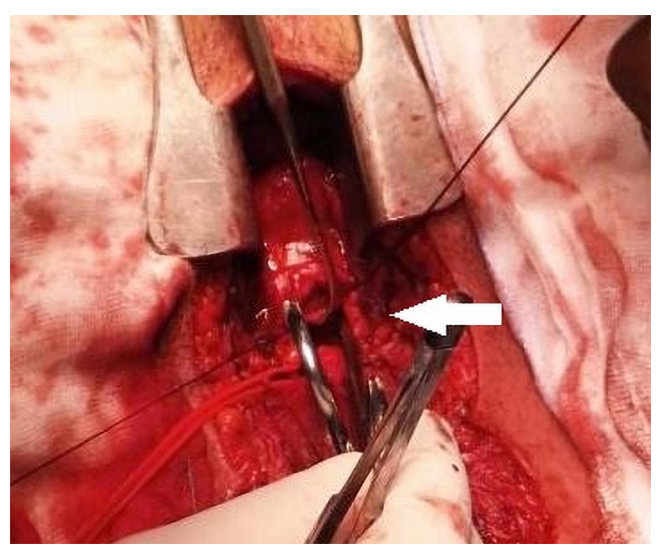

Figure 2 Intraoperative view: complete transection of the intrathoracic trachea (arrow).

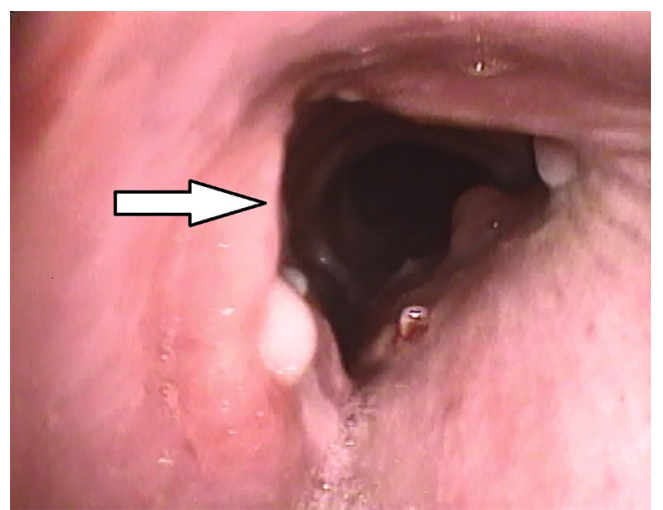

Figure 3 Tracheoscopy 30 days after surgery showing healed tracheal anastomosis (arrow). fluoroscopic guidance and transthoracic echocardiography, femoro-femoral veno-venous ECMO was instituted with a multistage drainage $25 \mathrm{Fr} / 38 \mathrm{~cm}$ HLS cannula (Maquet, Rastatt, Germany) in the left femoral vein and a reinfusion single stage $21 \mathrm{Fr} / 55 \mathrm{~cm}$ Biomedicus cannula (Medtronic, Minneapolis, USA) in the right femoral vein. Cannulas were navigated towards the lower IVC at the level of the renal veins and the right atrium (RA), respectively. A PLS Maquet circuit was connected and started at $3.3 \mathrm{~L} / \mathrm{min}$ $(2,200 \mathrm{rpm})$ with $\mathrm{FiO}_{2} 1.00$ and sweep gas flow $2.5 \mathrm{~L} / \mathrm{min}$. During apneic ventilation pump flow was increased to $4.4 \mathrm{~L} / \mathrm{min}(2,800 \mathrm{rpm})$. No anticoagulation was administered, and the patient received four units of packed red blood cells, four units of fresh frozen plasma and 3 gr of fibrinogen over the course of surgery.

Tracheal repair was performed through a cervicotomy and partial upper sternotomy. The distal margin of the proximal tracheal stump was identified at the level of the left brachiocephalic vein, and the distal tracheal segment was reached $1 \mathrm{~cm}$ distally to the innominate artery. Following mobilization on the anterior tracheal plane, the tracheal stumps could be approximated without tension (Figure 2). End-to-end anastomosis with 3-0 interrupted re-absorbable suture was performed. The stitches on the posterior tracheal wall were tied and a $7.5 \mathrm{~mm}$ endotracheal tube was inserted through the glottis. The tube was then positioned under direct vision in the distal tracheal segment. Following completion of the anastomosis, a pedicled cervical strap muscle flap was interposed between the innominate artery and the tracheal anastomosis to prevent tracheo-innominate artery fistula. No periods of hypoxemia or significant bleeding were observed during the operation. After arrival in the ICU low dose anticoagulation with bivalirudin was started, targeted to aPTT 45-50 sec (aPTT P45 sec corresponding to a ratio $\geq 1.5$ ). The patient was extubated 24 hours after surgery and weaning from ECMO was accomplished on the fourth post-operative day, following improvement of the bilateral lung contusion. Endoscopic follow-up 30 days after surgery showed a healed tracheal anastomosis (Figure 3).

\section{Discussion}

In the present case a patient with traumatic intrathoracic tracheal transection impossible of intubation of the distal trachea was successfully treated by using venovenous ECMO during tracheal repair. However, possible 
alternative therapeutic approaches need to be discussed.

\section{Question 1: in this patient intubation of the distal tracheal stump could not be accomplished due to the risk of causing a migration of the distal segment into the mediastinum. Would an emergent surgical approach without the use of ECMO be a safe option?}

\section{Expert 1}

The trauma-team should be acknowledged for their wise decision to use bronchoscopy for airway management. Unless an emergency thoracotomy had been needed for immediate rescue, it is hard to see anything but extracorporeal support in the current case. Highest priority is to establish a patent airway (and spinal neck control), and second step to provide gas exchange. The airway was not safe and to meet goals mentioned with additional minimal risk of insult peripheral cannulation ECMO was appropriate. I might have considered VA ECMO but from the data given VV seems adequate. Low-flow extracorporeal methods are discarded since oxygenation support requires ECMO, i.e., blood flow $>2 \mathrm{~L} / \mathrm{min}$ (4).

In the following I will comment on cannulation approaches when jugular access is disqualified. Cannula choice and cannulation configurations are important in VV ECMO concerning recirculation, i.e., effectiveness of the support. The margins for drainage and reinfusion of oxygenated blood may also be compromised from compression/dislodgement of intrathoracic structures, and in worst case, development of thoracic compartment syndrome.

Awake cannulation with local analgesia (if needed careful titration of additional low dose s-ketamine, alpha 2-agonist or benzodiazepine) during maintained spontaneous ventilation seems to carry the least risk for pneumo-mediastinum etc. All kinds of positive pressure ventilation should be avoided till the distal part of the airway is secured. Broad spectrum antibiotics including antifungals should be administered. I would avoid epidural analgesia due to the anticoagulation to come. To prolong the time for anticoagulation free support all circuit components should be coated.

\section{Cannulation strategies}

In VV ECMO there is no 'by-pass', the only cardiac output (CO) there is, is the patient's CO. The net points for drainage and return are important in respect of recirculation, i.e., for a high effective ECMO flow $\left(\mathrm{Q}_{\mathrm{EFF}}\right)$ : $\mathrm{Q}_{\mathrm{EFF}}=\mathrm{Q}_{\mathrm{EC}} \times\left(1-\mathrm{R}_{f}\right)$, where $\mathrm{Q}_{\mathrm{EC}}$ denotes ECMO flow, and $\mathrm{R}_{f}$ recirculation fraction. Recirculation $(\mathrm{R})$ is defined as the proportion of ECMO flow that does not contribute to the patient's oxygenation, i.e., is drained back to the ECMO circuit without mixing with the genuine venous blood $(4,5)$. The distance between drainage and return should be as far as possible $(6,7)$. Best if separated to different venous blood streams. Drainage of the lowest saturated blood should always be the aim in any mode: VV, VA, VVA, for increased leverage of oxygen transfer over the ML $(7,8)$.

Veno-venous dual-lumen cannula approaches

The placement of a cavo-atrial dual-lumen cannula (DLC) (termed not to be feasible in the current case) would be high risk since drainage problems may develop from right atrium and superior vena cava (SVC) compression. Increased intrathoracic pressure or cardiac tamponade affects right ventricular and subsequent left ventricular $\mathrm{CO}$ resulting in reduced oxygen delivery, but also increase Rf, thus limiting oxygen delivery via the membrane lung (ML). Bi-caval DLC would allow for some extra margin since part of the drainage occurs in the IVC.

Veno-venous single lumen cannula approaches

(I) In the current case femoro-femoral configurations via the groin/s were applicable. Drainage would be preferred as described, i.e., distal to the return of oxygenated blood. The probability for $\mathrm{R}$ was low in this "lower IVC to upper IVC" configuration and $\mathrm{Q}_{\mathrm{EFF}}$ thus probably close to $\mathrm{Q}_{\mathrm{EC}}$. In case of increased intrathoracic pressures, venous return would be compromised and thus CO reduced unless compensated for by increased heart rate. Advancing the return cannula tip from the upper IVC to RA (intra-thoracic position) may increase the marginals in such case. The drainage cannula's position in the lower IVC favored a low Rf. However, in patients with intra-abdominal hypertension, abdominal compartment syndrome, or hypovolemia, pumping problems may occur due to drainage limitations. The patient will need volume.

(II) In femoro-jugular single lumen cannulation (SLC) oxygenated blood enters the RA via the SVC. Oxygen delivery is maintained according to patient CO. The same physics as in the example above where the femoral return cannula was advanced into the RA prevail.

(III) Jugulo-femoral SLC with drainage from the SVC/ $\mathrm{RA}$ and return to the femoral/iliac vein requires cannulation at the neck. Mediastinal/intrathoracic pressure may compress the SVC and cannulation may be hard or impossible. If occurring after commencement of ECMO drainage may be severely 
affected. Return flow from the IVC would cease. A long femoral lighthouse tip, "single-stage", cannula could be advanced up to the lower SVC. If a multistaged cannula is used in such configuration the drainage will not occur close to the tip but rather at the most proximal rows of holes of the drainage cannula (10-20 cm from the tip) $(5,7)$. Since return occurs distal to this $\mathrm{Rf}$ may be $100 \%$, i.e., no oxygenation support to the patient.

(IV) In case of inappropriate drainage flow, a SLC approach using two drainage cannulae may be applied: one placed in the IVC distal to the return cannula and one at the junction of SVC/RA (via the jugular vein, femoro-jugulo-femoral, or "low IVC-SVC to upper IVC", VV-V configuration). In this case the return cannula should be placed in the upper part of the IVC. If placed too low in the IVC recirculation is likely if venous return decreases. On the other hand, if placed too far up (RA), Rf may be high since the return and drainage (SVC/RA) are close. This problem would be exaggerated if drainage via the lower cannula decreases of any cause. If reduced drainage from the upper cannula, drainage would part bed compensated for by the IVC cannula. To use a long multi-staged interrupted cannula designed to drain both at the tip (SVC) and at a perforated sequence $15-20 \mathrm{~cm}$ further down the cannula stem may be deceiving since, at lower flows, most drainage will occur at the latter site (as long as the holes not cling to the vascular wall) $(5,7)$. There will be a lot of 'traffic' in the IVC since the return cannula must be in the RA (for low R). In any case, drainage problems have to be recognized and measures taken. The cause might be of lifethreatening character, e.g., intra-thoracic bleeding, pressure-pneumothorax, intra-abdominal bleeding or compartment syndrome, etc.

\section{Veno-arterial approaches}

Peripheral VA configuration would be the choice if CO decreases (cardiac failure) leading to/risk to lead to insufficient oxygen delivery during resuscitation. VA may also be introduced in trauma cases with severe (venous) bleedings since the central venous pressure decreases and the 'deadly' triad of hypothermia, coagulopathy and acidemia may get controlled. Measures should also be taken to assess right ventricular function if pulmonary vascular resistance increases.

(I) In femoro-femoral VA ECMO blood is drained from the IVC with return in the iliac artery via the groin. This is a feasible configuration when lung function is in balance with cardiac function for adequate for oxygenation of the upper body. Differential hypoxemia is always present in peripheral cannulation VA ECMO (7-9). It should be realized that there is a continuous risk to development fulminant differential hypoxemia $(\mathrm{FDH})$ in VA with IVC drainage if the heart recovers before the lung. If native $\mathrm{CO}$ pushes the mixing zone (where the retrograde ECMO flow mixes with blood ejected by the left ventricle) below the diaphragm, i.e., down to the abdominal aorta, no extracorporeal oxygen will enter the heart driven "upper circuit" in the body. The ECMO blood perfuses the lower body tissues and that venous blood, still rather high in oxygen content will be drained out to the ECMO circuit via the drainage cannula (in the IVC). The only oxygen entering the upper body's circulation will be via the sick lung. If oxygen consumption is higher than what the lung may contribute with end-organ (coronaries, brain, etc.) hypoxia is an imminent threat. If $\mathrm{SaO}_{2}$ in the upper body is lower than $\mathrm{SpreO}_{2}$, consider immediate intervention. Most centers today convert to $\mathrm{V}-\mathrm{AV}$ configuration, see (III) below. Conversion for VV ECMO should be discussed, but consider pulmonary hypertension and right ventricular dysfunction/failure. Best intervention, (requires access to the neck though), would be to apply a jugulo-femoral VA configuration (II).

(II) Jugulo-femoral VA ECMO with drainage from the SVC/RA and return in an iliac artery is the safest SLC configuration to avoid FDH and keep the ECMO circuit free from unnecessary connectors etc. $(10,11)$. Drainage via a jugular approach for high flows is recommended but is also possible via a femoral vein using a long 27-29 Fr lighthouse tip cannula positioned in the SVC/RA. The risk is venous stasis of the cannulated leg and secondary deep venous thrombosis. By drainage form the SVC (e.g., jugular multi-stage, $25 \mathrm{Fr} / 38 \mathrm{~cm}$ HLS used for IVC drainage in the current case), high flow of the least saturated blood in the body is permitted. The low venous oxygen content allows for high oxygen delivery over the ML. After return of the oxygenated blood in the iliac artery the lower body in perfused in retrograde direction. Still on the venous side (IVC) the oxygen content is rather 
high $\left(\mathrm{SO}_{2} 80-85 \%\right)$. The volume-fraction of the desaturated venous blood drained from the SVC will, according to the Frank-Starling curve as long as $\mathrm{CO}$ is maintained, be replaced by venous return to the RA by the highly saturated blood from the IVC. Oxygen saturation in the pulmonary artery increases, lung function or not, end-organ oxygen delivery in the upper body increases.

(III) Applying V-AV by introduction of a venous return cannula to increase oxygen content in the upper body/'circuit' in femoro-femoral VA ECMO solves the FDH situation (9). However, it does not correct the basic problem of a drainage cannula in the wrong position. Additional connectors/Y-pieces and flow regulation devices needed to divert and balance the respective $\mathrm{VV}$ and VA flows cause turbulence and increased shear stress which may promote activation of coagulation, hemolysis, etc. (12).

(IV) Return cannula placement in a chimney graft on the subclavian or innominate arteries has been described $(13,14)$. These approaches should not be considered in the critically ill/trauma patients but rather for bridge to transplant and will not be further discussed here.

\section{Expert 2}

The impossibility to intubate the distal tracheal segment prevented from obtaining a secured airway, that means the presence of a cuffed endotracheal tube below the vocal cords. The success of laryngeal mask in restoring oxygenation should be considered an effective emergent measure, but not a safe solution allowing for a surgical operation on the thorax with a consistent risk of intraprocedural loss of airway control. On these premises, it is my opinion that in this case the use of mechanical support was mandatory to ensure adequate respiratory exchanges before proceeding to operation.

\section{Question 2: due to the multiple trauma and the inberent risks of surgical bleeding, anticoagulation was started only two hours postoperatively in order to safely assess coagulation parameters and surgical issues. Which could have been the best approach concerning intra- and postoperative anticoagulation while using ECMO?}

\section{Expert 1}

Intra-operative approach would be to have a balanced coagulation not using thrombin inhibitors or heparin.
Careful hemostasis should be obtained by using cautery and to wait a few minutes and do a secondary assessment of bleeding control before closing the wound, or to use a vacuum dressing. A few hours after surgery till "after-night" (depending on signs of circuit fibrin deposits etc., and coagulation labs) and bleeding control kept, anticoagulation should be started without any boluses, and signs of bleeding/circuit clotting monitored accordingly.

\section{Expert 2}

Delayed anticoagulation in patients on veno-venous and veno-arterial ECMO has been described and can be considered as a feasible option in the case of ECMO during surgery or shortly after surgical operation. In this case, the multiple traumatic intrathoracic lesions put the patient at a very high risk of bleeding; therefore, it is an absolutely reasonable approach to run ECMO without anticoagulation, reserving its start for the postoperative period.

\section{Question 3: could cardio-pulmonary by-pass have been a viable option in the treatment of this post-traumatic tracheal lesion?}

\section{Expert 1}

Every center works in its own context of culture, experience, available resources, physicians' and nursing staff's comfort zone. Maybe central cannulation would be the best at some institutions. However, central cannulation "ECMO" carries higher risk for bleeding and later infections. Bypass might be "over-doing" if not required for support of CO if there only is the temporal problem with airway patency. Other limiting factors for bypass are risk for cerebral embolization. The benefit would be maintained oxygenation of the brain in case of loss of lung function.

\section{Expert 2}

$\mathrm{CPB}$ has been used for tracheal surgery and to safely perform surgical operation not involving heart or great vessels but burdened with a high risk of hypoxemia or hemodynamic instability. CPB is associated to: absolute need for full systemic heparinization; higher level of coagulation system activation due to the presence of air-blood interface and larger area of contact between blood and artificial surfaces; higher risk of postoperative hemorrhage. Moreover, the use of $\mathrm{CPB}$ does not allow the continuation of mechanical support to hasten the weaning from mechanical ventilation. In my opinion, CPB for posttraumatic tracheal surgery should be reserved for cases with 
concomitant heart or great vessels injuries.

\section{Conclusions}

Post-traumatic tracheal repair is usually performed with the use of cross-field intubation or high-frequency jet ventilation $(1,15)$. However, when emergent intubation of the distal tracheal stump is not possible, other options have to be considered to maintain adequate gas exchange during surgical treatment to prevent the risk of losing pulmonary ventilation once the mediastinum is exposed.

The use of veno-venous or veno-arterial ECMO has been described during complex tracheal and lung surgery for benign and malignant airway lesions in pediatric and adult patients $(3,16)$. However, the use of ECMO in the treatment of post-traumatic lesions has been rarely described (17). Advantages with the use of ECMO during tracheal surgery include the possibility of avoiding the use of cross-field ventilation with endotracheal cuffed tubes or high-frequency jet ventilation catheters, improving visualization of the surgical field and preventing periods of intermittent ventilation that may be required during surgery. In patients with major pulmonary contusion this point may represent a significant advantage to maintain adequate gas exchange during surgery. In the treatment of post-traumatic airway lesions ECMO may have some advantages over $\mathrm{CPB}$, as the possibility of reducing the need for anticoagulation thanks to the increased biocompatibility with coating in ECMO systems, a major point in trauma patients. In the present case anticoagulation was started only postoperatively in order to reduce the risk of bleeding during surgery. Early extubation after surgery may also be accomplished, preventing the need for postoperative high-pressure ventilation and favoring healing of airway repair. Veno-venous ECMO is an effective way of maintaining adequate gas exchange during surgery, while the use of veno-arterial ECMO may be required to maintain hemodynamic stability as when mobilization of the heart is required during surgery. The advantages of the use of ECMO have however to be weighed against potential complications as vascular lesions due to cannulation, bleeding and thromboembolism (18). Some technical details deserve attention when considering this strategy: first, the neck is completely non-approachable either for cannulation and for transesophageal echocardiography, making femorofemoral cannulation mandatory; second, ECMO should not be removed immediately after surgery, rather fastening weaning from mechanical ventilation reducing airway and lung barotrauma.

In selected patients with post-traumatic complex tracheal lesions with critical airway control and impaired respiratory function ECMO may be a life-saving option to allow adequate gas exchange and urgent surgical treatment of the airway lesion.

\section{Acknowledgements}

None.

\section{Footnote}

Conflicts of Interest: The authors have no conflicts of interest to declare.

Informed Consent: Written informed consent was obtained from the patient for publication of this manuscript and any accompanying images.

\section{References}

1. Mathisen DJ, Grillo HC. Laryngotracheal trauma. Ann Thorac Surg 1987;43:254-62.

2. Wiebe K, Baraki H, Macchiarini P, et al. Extended pulmonary resections of advanced thoracic malignancies with support of cardiopulmonary bypass. Eur J Cardiothorac Surg 2006;29:571-7.

3. Lang G, Ghanim B, Hötzenecker K, et al. Extracorporeal membrane oxygenation support for complex tracheobronchial procedures. Eur J Cardiothorac Surg 2015;47:250-5.

4. Conrad SA, Broman LM, Taccone FS, et al. The Extracorporeal Life Support Organization Maastricht Treaty for Nomenclature in Extracorporeal Life Support. A Position Paper of the Extracorporeal Life Support Organization. Am J Respir Crit Care Med 2018;198:447-51.

5. Palmér O, Palmér K, Hultman J, et al. Cannula Design and Recirculation During Venovenous Extracorporeal Membrane Oxygenation. ASAIO J 2016;62:737-42.

6. Abrams D, Bacchetta M, Brodie D: Recirculation in venovenous extracorporeal membrane oxygenation. ASAIO J 2015;61:115-21.

7. Lindholm JA. Cannulation for veno-venous extracorporeal membrane oxygenation. J Thorac Dis 2018;10:S606-S612.

8. Frenckner B, Broman M, Broome M. Position of draining venous cannula in extracorporeal membrane oxygenation 
for respiratory and respiratory/circulatory support in adult patients. Crit Care 2018;22:163.

9. Choi JH, Kim SW, Kim YU, et al. Application of venoarterial-venous extracorporeal membrane oxygenation in differential hypoxia. Multidiscip Respir Med 2014;9:55.

10. Hou X, Yang X, Du Z, et al. Superior vena cava drainage improves upper body oxygenation during veno-arterial extracorporeal membrane oxygenation in sheep. Crit Care 2015;19:68.

11. Lindfors M, Frenckner B, Sartipy U, et al. Venous Cannula Positioning in Arterial Deoxygenation During Veno-Arterial Extracorporeal Membrane Oxygenation-A Simulation Study and Case Report. Artif Organs 2017;41:75-81.

12. Fuchs G, Berg N, Broman LM, et al. Flow-induced platelet activation in components of the extracorporeal membrane oxygenation circuit. Sci Rep 2018;8:13985.

13. Biscotti M, Bacchetta M. The "sport model": extracorporeal membrane oxygenation using the subclavian artery. Ann Thorac Surg 2014;98:1487-9.

14. Chicotka S, Rosenzweig EB, Brodie D, et al. The "Central Sport Model": extracorporeal membrane oxygenation using the innominate artery for smaller patients as bridge to lung transplantation. ASAIO J 2017;63:e39-e44.

15. Carretta A, Melloni G, Bandiera A, et al. Conservative and surgical treatment of acute posttraumatic tracheobronchial injuries. World J Surg 2011;35:2568-74.

16. Hines MH, Hansell DR. Elective extracorporeal support for complex tracheal reconstruction in neonates. Ann Thorac Surg 2003;76:175-8.

17. Enomoto Y, Watanabe H, Nakao S, et al. Complete thoracic tracheal transection caused by blunt trauma. J Trauma 2011;71:1478.

18. Rinieri P, Peillon C, Bessou JP, et al. National review of use of extracorporeal membrane oxygenation as respiratory support in thoracic surgery excluding lung transplantation. Eur J Cardiothorac Surg 2015;47:87-94.

Cite this article as: Carretta A, Ciriaco P, Bandiera A, Muriana P, Pappalardo F, Broman LM, Montisci A, Negri G. Veno-venous extracorporeal membrane oxygenation in the surgical management of post-traumatic intrathoracic tracheal transection. J Thorac Dis 2018;10(12):7045-7051. doi: 10.21037/jtd.2018.11.117 